\title{
Schema Therapy and the use of Politeness Plural in Greek-speaking populations: a need for cultural adaptation or a quest for early maladaptive schemas/modes?
}

\author{
Konstantina Kolonia $^{1}$, Eirini Tsartsara ${ }^{2}$ and Ourania Giakoumaki ${ }^{3}$ \\ ${ }^{1}$ West London Mental Health NHS Trust, Hounslow Recovery Team East, 729 London Road, \\ London TW3 1SE \\ ${ }^{2}$ Greek Society of Schema Therapy, Sisini 17, 11528, Athens, Greece \\ ${ }^{3}$ El. Venizelou 14-16 Argyroupoli, Athens, Greece
}

Received 29 March 2018; Accepted 21 November 2018

\begin{abstract}
This paper is clinical practice-based, and examines the Greek cultural linguistic schema of Politeness Plural in the application of the Schema Therapy mode model. The philosophical principles of Schema Therapy and the importance the model ascribes on creating a warm therapist-client relationship as a pre-requisite for schema healing are discussed. We further explore the need for the therapist to be culturally sensitive to the linguistic use of Politeness Plural in Greek-speaking populations. We are looking into the reasons of why, whilst culturally sensitive, a schema therapist needs to remain inquisitive of potential maladaptive and/or internalized dysfunctional coping mechanisms of inter-relating that are masked by the use of the Politeness Plural linguistic schema. The implications the cultural linguistic schema of Politeness Plural can have in the therapy outcome are considered. Specifically, we argue that holding on to the Politeness Plural cultural linguistic schema may reinforce emotional distancing and compromise schema healing. The authors identify the need for more research to further understand the issues that are raised in this article. Although the focus of the article is on Greek populations, the matters under consideration may be valuable to other cultures.
\end{abstract}

Key words: cultural adaptation, culturally sensitive, evidence-based practice, personality disorder, schema

\section{Introduction}

Cultural schema theory suggests that the experiences that are unique to individuals allow them to acquire personal schemas (Sharifian, 2008; Sharifian and Zamarani, 2011). Specifically, cultural schemas are conceptual structures that enable individuals to store perceptual and

Author for correspondence: Konstantina Kolonia, West London Mental Health NHS Trust, Hounslow Recovery Team East, 729 London Road, London TW3 1SE (email: konstantina.kolonia@wlmht.nhs.uk).

(C) British Association for Behavioural and Cognitive Psychotherapies 2019 
conceptual information about their culture and interpret cultural experiences and expressions (Bernal et al., 2009). Therapeutic models are culturally adapted in different countries. Bernal et al. (2009) have developed a framework for enhancing cultural ecology when adapting psychotherapy for treatment outcome research. They described eight overlapping cultural dimensions and features of the treatment population to incorporate into psychotherapy outcome research: concepts, language, persons, content, metaphors, goals, methods and context. The current article focuses on language (e.g. as the main vehicle of expression within one's culture) as one of the cultural dimensions and features informing one's cultural schema. Specifically, we are discussing the Greek cultural linguistic schema of Politeness Plural in the application of the Schema Therapy (ST) Mode model.

\section{Politeness phenomena in English and Greek language}

In medieval and early modern European languages, honorific systems were more elaborate. Societies were more clearly stratified then and people conscientiously followed the patterns of language, which supported class divisions (Sifianou, 2008). English language had a T/V distinction (from the Latin pronouns $t u$ and vos) as follows: thou and ye for the second person nominative singular and plural, and thee and you for the second person accusative singular and plural, respectively. The plural you was then generalized as nominative for both singular and plural. It was promoted to cover both usages, thus enhancing the distance between the speakers and keeping class restrictions where they should be. Nowadays you doesn't have the same distancing connotations (Sifianou, 2008).

On the other hand, the Greek language includes a pronominal system similar to those of many European languages. The second person plural (Eseis-You) can be used towards a single recipient as a means of indicating formality and/or politeness either because of status differences or social distance between the contributors in an encounter (Sifianou, 2008). When using the second person to an individual in Greek, a choice must be made between using the singular or plural form of the verb. The choice made depends upon the relationship between the talker and the person he is referring to. The singular form (Esy-You) is familiar and informal, used with family, friends, children and younger people. The plural (Eseis-You) is formal and polite, and used with strangers and to give respect.

Besides the common Kirios-Mr, Kiria-Ms titles that are the most distancing and impersonal, forms of address include those of occupation or position and respect. Such an example of occupational or positional titles (e.g. refer by title judge, lawyer, Dr, etc.) in psychotherapy is the use of jatre-Doctor to address psychiatrists/psychologists (Sifianou, 2008).

Therefore, the cultural linguistic schema of Politeness Plural (e.g. to achieve positive politeness/formality for the speaker) underlies the enactment of several speech acts in Greek (e.g. expressing gratitude, respect to older people or figures of authority and/or creating distance amongst the speakers) and is adopted by the majority of the practising psychotherapists.

\section{Why the need for cultural adaptation?}

As three Greek-speaking psychologists educated in the United Kingdom and having worked with Greek and non-Greek populations across Europe, we quickly became very mindful of how crucial it is that the therapist is culturally sensitive to the population she is working with 
for therapeutic interventions to be efficacious. What do we mean though, when we say that we are working in psychotherapy with models that are culturally sensitive? Cultural adaptation refers to the process of making psychotherapy goals, content, language and process consistent with those of the target population to enhance treatment credibility, efficacy and relevance (Sharifian and Zamarani, 2011). Cultural adaptation of psychological treatments is important because many evidence-based psychological models have been developed and tested mainly among Caucasian middle-class Americans (Koslofsky and Rodríguez, 2017).

Within our roles as supervisors and therapists of Greek-speaking populations in the Schema Therapy model (Young, 1999), we have been called to face the following questions: Is it always necessary to adapt to the culture blindly for therapy to be efficacious? Do we, as therapists, need, whilst being culturally sensitive, to be inquisitive to the extent of even challenging cultural linguistic schemas?

\section{Schema Therapy objectives, therapists' stance and the challenges for the Greek schema therapist}

ST is an integrative, innovative and evidence-based psychotherapy that was developed by Jeffrey Young (1999) for challenging and complex patients. Those patients often present with vague yet pervasive and chronic problems, difficulties in establishing and maintaining relationships, and highly avoidant and rigid patterns of coping (Edwards and Arntz, 2012; Young et al., 2003). ST focuses on unmet core emotional needs in childhood and the development of self-defeating, self-perpetuating and resistant to change emotional and cognitive patterns known as early maladaptive schemas (Young, 1999).

For the more complex presentations such as borderline and narcissistic personality disorders, the schema mode concept is introduced (i.e. it reflects the emotional and behavioural state at a given moment in time in an individual, and consists of thoughts, emotions and behaviours) and is now seen as the essence of Schema Therapy for those populations. Young et al. (2003) have proposed ten schema modes that fall into four categories:

(1) Child modes (Vulnerable, Angry, Impulsive/Undisciplined, and Happy) that are believed to be innate and universal and develop when specific core emotional needs are not met in childhood.

(2) Maladaptive Coping modes that correspond directly to the three coping styles of Overcompensation, Avoidance and Surrender.

(3) Dysfunctional Parent modes (Punitive, Demanding /Critical) that mirror the internalized behaviour of the parents towards the patient as a child.

(4) Healthy Adult mode, which is the functional part of the self.

The goal of Schema Mode Therapy is to help the patient achieve and remain in this mode so as to nurture, protect, and/or set limits at the Child parts when these are triggered by day-today life experiences. It further aims to combat the unhealthy messages from the Dysfunctional Parent modes and help the patient to develop healthier coping strategies and ways of relating to self and others (Young et al., 2003).

The therapist-client relationship is vital for schema healing. Limited re-parenting and empathic confrontation are key relational tools (Kolonia and Kyritsi, 2017). The process of limited re-parenting is the heart of the treatment and one of its most unique and defining elements. It refers to the overall stance and style of a therapist who is optimally acting from 
a Healthy Adult/Good Parent mode (Farrell et al., 2014). His/her primary objective is to attune and achieve emotional connection, access and heal the wounds and unmet needs of the patient's Vulnerable Child self/mode - before achieving unification of conflicting internalized dysfunctional parent and maladaptive coping modes/parts of self. On the other hand, empathic confrontation describes a therapist stance that integrates an understanding and validation of the patient's experience at the same time as emphasizing a need for change (Young et al., 2003).

These two concepts of limited re-parenting and empathic confrontation distinguish ST from other psychotherapeutic approaches. ST requires a therapist, whose 'language', both verbally and non-verbally, conveys: warmth, directness, openness, intimacy, sharing of emotions within the relational context, flexibility, limit setting, and self-disclosure, whenever appropriate. In traditional forms of psychotherapy in Greece, where the therapist aims to stay neutral and not encourage dependency, the Politeness Plural (e.g. Mr/Ms) or occupation/positional titles (Doctor) are favoured.

In ST, 'the process of limited re-parenting involves welcoming and encouraging this dependency. The therapist's regulation of the patient's affect becomes internalized by the patient and forms a healthy adult mode modelled on the therapist's. The healthy adult mode becomes a strong foundation for the establishment of autonomy' (Lockwood, 2008).

The challenge posed in applying ST in a Greek-speaking psychotherapy system, where the client usually expects to be addressed and addresses his/her therapist in second person plural, is that ST utilizes a vast array of emotion-focused techniques in which the therapist is best to address the age-wise little Vulnerable Child mode of the client in second person singular in order to be consistent with the therapy concepts presented earlier.

For example, in imagery re-scripting when the therapist is called to re-parent the Child mode, she invites her client to imagine her talking to their Child part as a good enough parent would do. Similarly, when the therapist aims to set limits or stand up to the internalized parent modes of the client they will do it as a caring, protective, good enough parent would do. We are therefore wondering whether the well-evidenced effectiveness of the model is then compromised if the therapist:

(a) addresses the age-wise little Vulnerable Child in second person plural (e.g. 'I would like to talk to you Little Ms Katia' instead of 'I would like to talk to you little Katia'.

(b) addresses the Vulnerable Child's Punitive/Abusive parent in second person plural rather than in second person singular (e.g. 'Mrs Jane I would like to tell you (formal you) that you are hurting little Ms Katia', instead of 'Mother/or Jane, I would like to tell you (informal you) that you are hurting little Katia').

The argument we are making here is not that the parent needs to be disrespected, but rather to be addressed by an adult who asserts mutuality, equality and respect. As the Dysfunctional Parent modes are internalized by the client, they 'exert' great power and are sometimes experienced and represented as frightening or guilt-inducing. We therefore, feel that the use of Politeness Plural might disempower the reparative role of the therapist in such a situation. On a similar note, in the first example a parent would have never addressed her/his child in the second person plural when they were caring for and nurturing them.

The points we have just drawn regarding the usage of T-distinction in the ST practice within a Greek-speaking culture, might be more in line with the philosophical premises of the approach, yet by no means are a rule of thumb. From our personal experience, both as 
therapists and supervisors of schema therapists, we are aware of instances that Politeness Plural is being used within a ST relationship either by the therapist or by the client, or by both. Our clinical experience suggests that variations in the usage of T-V distinction within the therapeutic dyad may sometimes be diagnostic of the maladaptive coping mechanisms of the client and/or of the therapist when the therapeutic relationship 'seeks' closeness and intimacy to be the vehicle for change.

Therefore, we aim to further discuss and explore to what extent the I-you linguistics within ST should be culturally adjusted or at what times the Greek linguistic standards of T-V distinction should be challenged to promote healing in line with the principles of ST.

\section{The use of Politeness Plural by the Greek-speaking schema therapist: cultural norm or the therapist's own early maladaptive schemas/modes?}

At the initial stages of therapy the client is being introduced to the philosophy of ST and the techniques that are going to be utilized. The client is also exposed to the ST language that they will be using together to get a mutual understanding of the client's presenting difficulties.

Being aware of the cultural linguistic schema of Politeness Plural, a schema therapist working with Greek-speaking populations asks her client how the latter feels about addressing each other using second person plural and makes the suggestion that, if this feels right for both of them, they could utilize second person singular, reciprocally. This, from our point of view, is crucial to be discussed in the first sessions. It will be more coherent with then applying ST techniques and particular visual imagery (e.g. re-parenting the Vulnerable Child) without needing to shift in the T-V distinction within the same session. We feel that it would otherwise be confusing for both therapist and client, and probably counter-therapeutic, particularly when the client is using a resisting Detached Protector Mode that holds her back from sharing openly personal emotions and painful experiences.

Drawing from our own clinical experience to date, when clients we worked with preferred to keep addressing their therapist in second person plural, this was usually a display of a Detached Protector Coping mechanism or of an internalized Demanding Parent mode. Hence a means for keeping a 'safe' distance from feeling more intimate with the therapist and/or of 'protecting' internalized parental/cultural messages of 'appropriateness towards others'.

As clinical supervisors in ST, we have often encountered examples where the supervisee therapist herself rather than the client feels 'safer' or feels the need to utilize and address her clients in second person plural. In such cases, two issues are explored (a) whether this is the therapist's overall style, influenced by other schools of therapy practice and (b) whether sustaining the Politeness Plural is being felt as 'safer' in specific therapist-client relationships due to the therapist's own early maladaptive schemas/modes being triggered by some client groups more than others.

(a) Even though rarer, regarding the therapist's overall style, we have encountered two different reasons why Politeness Plural may be preferred in general. One is that she feels 'safer' in using Politeness Plural in general, particularly if she is at the beginning of her psychotherapy practice. It appears that Politeness Plural as a means of addressing the I-You relationship is most of the time represented as an appropriate way of setting 'therapeutic barriers' and a means of not becoming 'too friendly' with the client. The other reason is that she feels it is more 'appropriate' (e.g. in line with the linguistic culture of Greek psychotherapeutic tradition). In the latter, the therapist, despite keeping Politeness Plural 
throughout the sessions, moves from the V- to T-distinction only when she is using imagery techniques for re-parenting and re-scripting. In exploring this through supervision, it appears that the therapist may be influenced by her own psychotherapy experience of another school of thought that is using Politeness Plural. She has learnt to address her own therapist and being addressed as a client in plural, and she is modelling the same framework in her own practice. This is understandable if we take into consideration the linguistic usages of T-V distinction in the Greek culture, and even more the fact that some therapists have experienced the T-V distinction from their own psychotherapy as a model.

(b) In most of the instances, however, it appears the therapist is avoiding introducing second person singular in the I-You relationship due to their own schemas/modes being triggered by some clients more than others. These clients share certain characteristics and are considered to be:

(i) Clients who may either present 'grandiose', devaluing and aggressive or, otherwise, 'too needy' and 'over-involving' as their main coping repertoire of relating to significant others.

The first characteristic is pertinent to the personality structure of the client. Clients having devaluation as their main coping mechanism, when relating to significant others, may appear dismissive overall of emotions and close relationships, 'too bossy' at times, or 'overcontrolling' within therapy. Understandably enough, this has to do with early experiences of usually mistrust and avoidant attachment patterns that they developed out of a need to protect themselves and remain as much connected as possible from a 'safe' position with significant others (Wallin, 2007). Although a schema therapist can understand where it all comes from, it may at times feel unpleasant or impossible to get close to when feeling intimidated by a client who is resorting to such overcompensation mechanisms.

With regard to the use of Politeness Plural, at least one of us - like many of our supervisees has encountered feeling initially defensive towards a dismissive client. Her immediate thought was to hold on to the V-distinction as a means of keeping a 'safe enough distance', setting an external 'barrier' between herself and her client out of fear of being intimidated by the latter. In this case, avoiding introducing the usage of second person singular within the ST framework appeared to only strengthen the therapist's own Detached Protector mode. We feel, the Politeness Plural in this, and similar cases, could only strengthen a dismissive and/or avoidant stance of the therapist over her client's maladaptive coping mechanism and not assist healthy mutual sharing of emotions when things get difficult in the intimate dyad.

Similarly, we have encountered supervisee therapists, who due to a presenting 'neediness' from their client to get too close and/or some 'intrusion' by asking too personal questions to the therapist; the latter holds on to Politeness Plural as a means of protecting herself from experiencing a violation of one's own personal space (e.g. due to her own difficulty to set the limits otherwise) or feelings of inadequacy (e.g. 'my client will find out I am flawed'). As such neediness is often a sign of quite traumatized clients, we would err on whether the usage of second person plural could assist the deeper connection needed by those clients too. At the same time, we feel that one does not need the use of Politeness Plural to set limits to an Impulsive Child mode of a client who might resort to too many out-of-session telephone calls or attempts to break the therapeutic barriers in any other way. The schema therapist (as a good enough parent) can model autonomy, respect, healthy functioning and set limits, whilst being 
warm and direct via utilizing second person singular both in addressing and being addressed by her client.

(ii) Elder-age wise - clients

Elder clients pose some dilemmas for all schema therapists, even those who mainly use second person singular as an overall style in their work. That is because the use of Politeness Plural is common practice in Greek linguistics for addressing an older person as a way of communicating to them your respect. However, we have noticed that when the issue is raised and explained in a sensitive manner, the elder client does not feel disrespected by being addressed in second person singular. In our experience, therapists have the need to hold on to the second person plural at times they experience elder clients as dismissive in their interpersonal relatedness. In such cases the client addresses the therapist in second person singular but refuses her therapist to do the same. Here, the therapeutic focus needs to be the over-compensatory coping mechanisms of her client that does not allow her to get close and build an intimate relationship of trust. Finally, an elder person's certain modes or ways of relating can sometimes be triggering to the schema therapist due to schemas and attachment patterns of interrelating with her own parent, such issues can again be discussed in supervision.

\section{(iii) Opposite sex clients}

The third characteristic is pertinent to the sex of the client. The intimate relationship that is being formed between therapist and client can trigger a client's feelings of erotic transference. Quite interestingly, in our experience being a younger female therapist and working with a male elder client, even though it could, does not seem to be always the issue, as erotic transference feelings can be triggered irrespective of age. Such issues can be worked through via building a warm and trusting therapeutic relationship in ST, that the safety of its context can allow exploration of any kind of emotions.

The conclusion one can draw from all of the above is, more often than not, that the insistence of the therapist to not abandon Politeness Plural may be due to personal fears. Fears of seeming 'too friendly', of experiencing 'intrusion in her privacy and not keeping appropriate limits', of 'being disrespected' and /or of feeling 'devalued' by her clients. Thus, Politeness Plural may turn, at times, into a mechanism of avoiding intimacy and closeness particularly when the therapist schemas of inadequacy, mistrust, failure, enmeshment or selfsacrifice, are triggered. When this is the case, early maladaptive schemas and experiences of the therapist can and need to be discussed in supervision, with the aim to assist her first in bypassing her own Detached Protector mode and/or expunging her Demanding Parent mode, in order to be able then to work through the relevant issues with her clients.

\section{The use of Politeness Plural by the Greek-speaking client: cultural norm or early maladaptive schemas/modes?}

Most of the clients, after an introduction to the ST principles, feel comfortable and able to abandon the linguistic Politeness Plural. Exception to the above are clients who are presenting from the initial consultation as 'overly friendly' and addressing the therapist using second person singular [something which, considering the linguistics of I-You between two initial 
strangers discussed earlier, is often diagnostic of a certain personality (emotionally unstable personality disorder/narcissistic personality disorder) traits].

When clients are asked how it would feel being addressed and addressing their therapist in second person singular within the therapeutic context, we have found some clients feel immediately relieved and welcome it, particularly if they are already experiencing their therapist as warm and caring and have felt comfortable within the therapy relationship from those first sessions. Others, despite feeling comfortable, may express ambivalence and disbelief that this could be even possible. Exploring this ambivalence in more depth, it appears that personal and/or cultural-linguistic schemas of Unrelenting Standards (e.g. 'A doctor is considered to be a figure of respect/power; she should only be addressed by using Politeness Plural') are hidden beneath; thus an idealization of the therapist could be reinforced if Politeness Plural is not challenged within the therapy relationship.

There is, however, a third category of clients who despite being invited to mutual second person singular, report that they feel fine being addressed in second person singular but uncomfortable with addressing the therapist reciprocally. We have observed that the insistence of clients to address their therapist using the Politeness Plural after a number of sessions, is usually indicative of specific difficulties that such clients are facing with intimacy and getting close to others. In addition, maladaptive coping and/or internalized Dysfunctional Parental mechanisms may be in operation and that needs to be further explored and addressed. Within this third category of clients insisting on addressing the therapist in second person plural, we have encountered clients with avoidant personality characteristics or with mixed narcissistic, obsessive compulsive and avoidant personality characteristics.

Our experience of dealing with clients of this third category, suggests that there can be a variety of reasons for keeping such a discourse pattern. Some clients we have worked with share a Detached Protector mode with underlying schemas of Mistrust/Psychological Abuse and Defectiveness/Shame that seem to trigger the mode each time. To them, holding on for a longer period of time to Politeness Plural, when addressing their therapist, was presented as a means of 'avoiding to de-idealize' the therapist; the fret of getting too close and being exposed was over-controlled via verbal attempts to take distance. Again, with empathy and confrontation for the maladaptive expression of their Detached Protector mode, they were able to overcome the obstacles and begin to address their therapists in second person singular.

\section{Discussion}

In this paper we raise an important - in our view - issue which we understand the Greek schema therapist is facing in everyday practice: namely, the challenges that the Greek linguistic schema of Politeness Plural poses for a therapeutic approach that calls for building a warm and informal - in terms of naming and addressing each other therapist-client relationship. We speculate that similar challenges might be a matter of concern to schema therapists who practise in cultures where Politeness Plural linguistic phenomena also exist (e.g. Spain or Germany where the T-V distinction is part of the everyday linguistic encounters). To our knowledge, there is no relevant literature on the issue. Thus, emanating from our personal knowledge and experience as Schema practising therapists and/or supervisors of colleagues who are trained in ST, all information and suggestions we are offering need to be looked at with caution as although they are evidence based, they do not follow a specific and systematic methodology. They rather consist of personal observations 
and are a first attempt to bring forward the issue of formal/informal form of address within the ST approach in a non-English-speaking culture.

In considering the usefulness of introducing the informal second person form of address reciprocally in a culture that embraces Politeness Plural linguistics in doctor-patient relationships, we need to keep in mind the differences of the ST approach with other psychotherapeutic schools. For example, psychoanalysis fosters a climate of formal address to enhance neutrality in the therapist's stance and assist transference phenomena (PerezSanchez, 2012). In the 1980s, Senger (1984) conducted a small survey in Massachusetts to explore naming practices in psychotherapy. Quite interestingly, psychologists as opposed to psychiatrists, and family system therapists, as opposed to other schools of practice, seemed to prefer or to resort to second person singular in addressing their client. There was also some evidence that even among psychoanalytically oriented psychotherapists of this study, five out of 14 practitioners chose to address their client by first name. Yet, there appeared to be a 'double standard' on how psychologists, psychiatrists and social workers in this survey preferred to be addressed. Even though the school of practice is not reported by the author, he states that around $15 \%$ of both psychologists and psychiatrists did use a double standard of naming, whilst social workers were more 'sensitive' to equality of naming.

Clearly, Senger's (1984) interesting findings suggest that the issue of formal vs informal ways of addressing each other in a therapeutic dyad merits further investigation. There is no doubt that different therapy approaches differ regarding the extent that they foster an informal as opposed to a formal address style between therapist and client. Still, it is worth investigating for example, whether such choices are influenced not just by school of thought of the practising therapist but also by her personality traits and/or by the personality make-up of the client.

Throughout this paper we clearly take the position that the Politeness Plural linguistic schema should be optimally bypassed by a Greek-speaking schema therapist who fosters closeness and reciprocity in the therapist relationship. Saying this, by no means would we like to suggest that an intimate therapeutic relationship of trust cannot be built within a therapeutic relationship that utilizes a formal linguistic schema of address. There are much more paralinguistic and non-verbal signs of expression of warmth, care and understanding by which a therapist can convey her feelings towards her client. Yet, we feel that using second person singular as a way of reciprocal address between therapist and client is more in line with the premise of ST regarding the overall stance of limited re-parenting and empathic confrontation of the therapist. The extent to which this truly fosters greater efficacy of the model in a Greekspeaking culture, or similar cultures that utilize the T-V linguistics, needs to be answered by relevant research.

For example, qualitative research might shed light on the experience, instances and reasons why a schema therapist might choose to not drop the Politeness Plural linguistic schema. Also a mixed methodology of qualitative and quantitative measures that explores Schemas and Modes of both therapists and clients might enhance understanding regarding the extent to which sustaining or not the Politeness Plural form of address may collude with certain Early Maladaptive Schemas or Maladaptive Coping Modes or Dysfunctional Parental Modes of either therapist and/or the client. In addition, treatment outcome research will be of significant value between clients who continue/choose to use second person plural form of address throughout their treatment and clients who embrace the second person singular and equally with therapists who continue to use the plural when they are using ST. 
Thus, future research on the topic is certainly needed before suggesting with certainty that adjusting the Politeness Plural linguistic to the ST model's premise would ensure greater efficacy of the ST model in a Greek-speaking culture.

\section{Conclusion}

Our experience of any attempt to hold on to the Politeness Plural cultural linguistic schema in the Greek-speaking culture employed by either the therapist or the client suggests that it reinforces emotional distancing and prevents the therapy working through those Maladaptive Coping and internalized Dysfunctional mechanisms of self that perpetuate schemas on both.

Considering the philosophy that underlies ST for building a warm, open and direct, in expression, relationship between therapist and client, schema therapists who embrace the Politeness Plural might need to re-adapt their style to reciprocal, informal and direct speech to their clients, particularly those who may suffer from lack of intimacy, spontaneity and trust in their close relationships or those who may avoid forming close bonds altogether.

Whilst the schema therapist needs to preserve her capacity to remain culturally flexible to the use of external markers of politeness, it is vital to be mindful and inquisitive to what extent the cultural linguistic schema of Politeness Plural is an attempt to cover up schema modes (e.g. a Detached Protector mode from the relationship; an internalized Demanding Parent that passes on the message of 'respect figures of authority') and/or maladaptive schemas (e.g. Entitlement; Defectiveness/Shame).

Although the cultural linguistic schema of Politeness Plural in the application of the ST mode model focused exclusively to our experience within the Greek-speaking population in this article, the issues we raised and discussed above may apply to other cultures with similar linguistic schemas.

\section{Main points}

(1) This paper is clinical practice-based and examines the Greek cultural linguistic schema of Politeness Plural in the application of the Schema Therapy mode model.

(2) The philosophical principles of Schema Therapy and the importance the model ascribes on creating a warm therapist-client relationship as a pre-requisite for schema healing are discussed.

(3) We further explore the need for the therapist to be culturally sensitive to the linguistic use of Politeness Plural in Greek-speaking populations.

(4) We look into the reasons of why, whilst culturally sensitive, a schema therapist needs to remain inquisitive of potential maladaptive and/or internalized dysfunctional coping mechanisms of inter-relating that are masked by the use of the Politeness Plural linguistic schema.

(5) The implications the cultural linguistic schema of Politeness Plural can have in the therapy outcome are considered. Specifically, we argue that holding on to the Politeness Plural cultural linguistic schema may reinforce emotional distancing and compromise schema healing.

(6) Authors identify the need for more research to further understand the issues that are raised in this article. Although the focus of the article is on a Greek population, the matters under consideration may be valuable to other cultures. 


\section{Acknowledgements}

This paper would not have materialized without the contribution of our supervisees and clients. We therefore wish to deeply thank them for inspiring us to address the topic.

\section{Conflicts of interest}

Konstantina Kolonia, Eirini Tsartsara and Ourania Giakoumaki have no conflicts of interest with respect to this publication.

\section{Ethical statement}

The authors have abided by the Ethical Principles of Psychologists and Code of Conduct as set out by the APA. No ethical approval was needed as the article is based on anecdotal clinical practice and hence personal experience of the authors. All examples discussed are anonymous and do not offend any relevant party. All statements of the present article represent the personal views of the authors and not their institutes of affiliation.

\section{Financial support}

This paper received no specific grant from any funding agency, commercial or not-for-profit sectors.

\section{References}

Bernal G, Jiménez-Chafey MI, Domenech Rodríguez MM (2009). Cultural adaptation of treatments: a resource for considering culture in evidence-based practice. Professional Psychology: Research and Practice 40, 361-368.

Edwards D, Arntz A (2012). Schema Therapy in Historical Perspective. In The Wiley-Blackwell Handbook of Schema Therapy: Theory, Research and Practice (eds M van Vreeswijk, J Broersen, M Nadort), pp. 3-26. Wiley-Blackwell.

Farrell JM, Reiss N, Shaw IA (2014). The Schema Therapy Clinician's Guide: A Complete Resource for Building and Delivering Individual, Group and Integrated Schema Mode Treatment Programs. Wiley-Blackwell.

Kolonia K, Kyritsi H (2017). Schema Therapy. In The SAGE Handbook of Counselling and Psychotherapy, 4th edn (eds C Feltham, T Hanley, L Winter). Sage Publications Ltd.

Koslofsky S, Rodríguez MMD (2017). Cultural adaptations to psychotherapy: real-world applications. Clinical Case Studies 16, 3-8.

Lockwood G (2008). Limited Reparenting: Limited Reparenting and Trusting Needs [online]. Available at: https://www.schematherapysociety.org/Limited-Reparenting

Perez-Sanchez A (2012). Interview and Indicators in Psychoanalysis and Psychotherapy. Karnac.

Senger HL (1984). First name or last? Addressing the patient in psychotherapy. Comprehensive Psychiatry 25, 38-43.

Sharifian F (2008). Cultural schemas in L1 and L2 compliment responses: a study of Persian-speaking learners of English. Journal of Politeness Research 4, 55-80.

Sharifian F, Jamarani M (2011). Cultural schemas in intercultural communication: a study of the Persian cultural schema of sharmandegi 'being ashamed'. Intercultural Pragmatics 8, 227-251. 
Sifianou M (2008). Politeness Phenomena in England and Greece: A Cross-Cultural Perspective. Oxford University Press.

Wallin DJ (2007). Attachment in Psychotherapy. New York: The Guilford Press.

Young JE (1999). Cognitive Therapy for Personality Disorders: A Schema-Focused Approach, 3rd edn. Florida: Professional Resource Press.

Young JE, Klosko JS, Weishaar ME (2003). Schema Therapy: A Practitioner's Guide. New York: The Guilford Press.

\section{Learning objectives}

(1) Language is a significant cultural dimension that needs consideration to ensure cultural ecology when adapting psychotherapy models in non-English-speaking cultures

(2) The Greek cultural linguistic schema of Politeness Plural poses certain challenges for the Greek schema therapist in the application of the Schema Therapy (ST) Mode model.

(3) Schema therapists and supervisors are called to consider when ST should be culturally adjusted and when cultural schemas on both therapists and clients should be challenged to promote psychological healing in line with the principles of ST. 